den Staat. Solche Staatsbediensteten spricht Depenheuer als neurotische Tabuerhalter wohl nicht an, da ja die staatliche Gemeinschaft dem Einzelnen erst Sinn geben soll. Und diesen Sinn nimmt wohl eher ein Amtsanwalt als ein Schwarzfahrer, der sich mit Kontrolleuren prügelt, an. Beide leben aber wohl in der gleichen Gesellschaft, ohne dass einer von Beiden auf ihren Untergang nennenswerten Einfluss hätte, was zumindest der Schwarzfahrer auch nicht haben will.

48 Josef Isensee, Verbotene Bäume im Garten der Freiheit, in: Otto Depenheuer (Hrsg.), Recht und Tabu, Wiesbaden 2003, S. 115, 137.

49 Otto Depenheuer, Das Bürgeropfer im Rechtsstaat, in: Ders. (Hg.), FS-Isensee Staat im Wort, Heidelberg 2007, S. 43, 60.

50 Ebd.

51 Dazu zum Beispiel die Untersuchungen: Hannah Arendt, Der Liebesbegriff bei Augustin, Berlin 2005, Kurt Flasch, Augustin - Einführung in sein Denken, Stuttgart 1980; Kurt Flasch/ Udo Reinhold Jeck (Hg.), Das Licht der Vernunft - Die Anfänge der Aufklärung im Mittelalter, München 1997.

52 Hans Kelsen, Gott und Staat, in: Ders., Aufsätze zur Ideologiekritik, Neuwied 1964, S. 29.

53 Hans Kelsen, Gott und Staat, in: Ders., Aufsätze zur Ideologiekritik, Neuwied 1964, S. 29, 30.

$54 \mathrm{Zu}$ diesem: Lawrence Wright, Der Tod wird euch finden - Al-Qaida und der Weg zum 11. September, München 2007.

55 Zur deren Ideologie: Matthias Küntzel, Djihad und Judenhaß: über den neuen antijüdischen Krieg, Freiburg 2003.

56 Carl Schmitt, Der Führer schützt das Recht - Zur Reichstagsrede Adolf Hitlers vom 13. Juni 1934, in: Positionen und Begriffe im Kampf mit Weimar-Genf-Versailles 1923-1939, Hamburg 1940, S. 199.

57 Hans Kelsen, Der Begriff des Staates und die Sozialpsychologie - Mit besonderer Berücksichtigung von Freuds Theorie der Masse, in: IMAGO 1922, S. 97, 139. In diesem Aufsatz wird anhand einer Auseinandersetzung gerade mit Durkheim gezeigt auf welchen psychologischen Fehlüberlegungen die Hypostasierung des Staates, Gottes und der Gemeinschaft beruht, ohne einem plumpen, ahistorischen Rationalismus zu verfallen.
58 Vgl.: Heinz Steinert/Helga Craemer-Schäfer, Straflust und Repression, Münster 1998, S. 211.

59 Michael Kilching, Opferschutz und der Strafanspruch des Staates - Ein Widerspruch?, in: NStZ 2002, 57, 59.

60 Dazu, dass es sinnvoller ist über die Herkunft der Strafe, denn über ihren Zweck nachzudenken, wenn man diese überwinden will, ist besonders Nietzsche eine unterschätze Referenz: vgl. Jochen Bung, Nietzsche über Strafe, in: ZStW, 2007, 130.

61 Zum Begriff: Peter Strasser, Verbrechermenschen - Zur kriminalwissenschaftlichen Erzeugung des Bösen, 2. erweiterte Auflage, Frankfurt 2005.

62 Gerhard Walentowitz, Mit flächendeckender Polizeikooperation in den Überwachungsstaat, in: Philipp Thiée (Hg.), Menschen Handel - Wie der Sexmarkt strafrechtlich reguliert wird, Berlin 2008, S. 183.

63 Dazu siehe insbesondere: Jochen Thielmann, Die Grenze des Opferschutzes, StV 2006, 41.

64 Dazu: Jochen Thielmann, Dialog mit dem unsichtbaren Dritten, in: Philipp Thiée (Hg.), Menschen Handel - Wie der Sexmarkt strafrechtlich reguliert wird, Berlin 2008, S. 259.

65 Ute Ingrid Haas/Olaf Lobermeier, Bürgerschaftliches Engagement in der Opferhilfe, Baden-Baden 2005, S. 12.

66 Carl Schmitt, Verfassungslehre, 1. Aufl. 1928, 8. Auflage, Berlin 1993, S. 235 .

67 www.staat-modern.de

68 Ute Ingrid Haas/Olaf Lobermeier, Bürgerschaftliches Engagement in der Opferhilfe, Baden-Baden 2005, S. 24 ff.

69 Ute Ingrid Haas/Olaf Lobermeier, Bürgerschaftliches Engagement in der Opferhilfe, Baden-Baden 2005, S. 32 ff.

70 Elias Canetti, Masse und Macht, München 29. Aufl. 2003, S 170 f..

71 Es ist aber auch nicht immer die stärkste Bastion des Humanismus und des Rechtsstaates. Dies veranschaulicht insbesondere seine Entscheidung vom 10.02.2004 zur Nachträglichen Sicherungsverwahrung, der durch das Gericht der Weg bereitet wurde.

\title{
Verwahrvollzug light?
}

\section{Zur Bewertung von Longstay-Einrichtungen als Ansatz zur Überwindung der Legitimationsprobleme der Sicherungsverwahrung ${ }^{*}$ \\ Von Tobias Mushoff}

\section{Die Renaissance der Sicherungsverwahrung}

Die Sicherungsverwahrung erlebt seit Mitte der 1990er in Deutschland eine beispiellose gesetzgeberische Extension, die sich auch dramatisch in der Zahl der Untergebrachten niederschlägt. Befanden sich im Jahr 1995185 Personen im Vollzug der Sicherungsverwahrung, hat sich die Population inzwischen mit 415 Personen im Jahr 2007 mehr als verdoppelt. ${ }^{2}$ Dies ist nicht das „Ende der Fahnenstange“. Nach neuesten Schätzungen ist mit einer weiteren Verdopplung der Zahl der Sicherungsverwahrten in den nächsten zehn Jahren zu rechnen. ${ }^{3}$ Es droht damit ein Rückschritt in die Zeiten vor der großen Strafrechtsreform.

Auch wenn das BVerfG der einfachen und der nachträglichen Sicherungsverwahrung mit seinen Entscheidungen aus den Jahren 2004 und 2006 bescheinigte, nicht (grundsätzlich) verfassungswidrig zu $\operatorname{sein}^{4}$, ist die Sicherungsverwahrung in ihrer derzeitigern Form weiterhin zu Recht massiver Kritik ausgesetzt. ${ }^{5}$ Einer ihrer neuralgischen Punkte ist seit eh und je ihr Vollzug.

Das in Deutschland geltende zweispurige Rechtsfolgensystem trennt zwischen Strafen und Maßregeln der Besserung und Sicherung, zu denen nach der Entscheidung des Gesetzgebers auch die Sicherungsverwahrung gehört. Nach dem Vollzug der schuldangemessenen Strafe kann die weitere Unterbringung nur noch als Sonderopfer zugunsten der Allgemeinheit gerechtfertigt werden. Dieses Sonderopfer ist aber den Betroffenen gegenüber nur zu legitimieren, wenn die Sicherungsverwahrung als ultima ratio auf der Grundlage einer dem Stand der Prognoseforschung entsprechenden - vor allem hinreichend breiten - Gefahrenprognose angeordnet wird, sich ihr Vollzug positiv vom herkömmlichen Strafvollzug abhebt und den Betroffenen die erforderliche Hilfe zuteil wird, um die Inanspruchnahme zugunsten der Allgemeinheit möglichst schnell wieder beenden zu können. Das „kalkulierte Risiko“ von Vollzugslockerungen ist zusätzlich auch in Zeiten einer erhöhten Sicherheitsorientierung einzugehen. ${ }^{6}$

Soweit die Theorie. Die Unterscheidung von Strafe und Maßregel findet aber bei der Ausgestaltung des Vollzugs der Sicherungsverwahrung im Gesetz und in der Rechtswirklichkeit zu wenig Beachtung. Bereits Kohlrausch bezeichnete die Differenzierung von Strafvollzug und Sicherungsverwahrungsvollzug als bloßen „Etikettenschwindel. “7 Dieser Vorwurf ist weiterhin häufig berechtigt. Die Sicherungsverwahrten werden weitgehend wie gewöhnliche Strafgefangene - allerdings oft mit weniger therapeutischen Angeboten - untergebracht. ${ }^{8}$

Während das BVerfG in einer frühen Entscheidung aus den 1950er Jahren wenig Sensibilität für die vollzuglichen Konsequenzen der Zweispurigkeit bewies, hebt das Gericht nun zu Recht hervor, dass zwischen dem gewöhnlichen Strafvollzug und dem Vollzug der Sicherungsverwahrung ein verfassungsrechtliches Abstandsgebot 
zu wahren sei. Damit ist gemeint, dass sich der Vollzug der Sicherungsverwahrung positiv vom herkömmlichen Strafvollzug abzuheben hat. ${ }^{9}$ Einige Kritiker der Sicherungsverwahrung wollten diesem Gebot bereits zuvor durch eine Transformation der heutigen Unterbringungssituation in einen „Hotelvollzug“ Rechnung tragen. ${ }^{10} \mathrm{Die}$ Diskussion, wie die vollzugliche Situation der Sicherungsverwahrten verbessert werden könnte, erhält durch die Einführung sog. Longstay-Konzepte mit freundlicher gestalteten Einrichtungen für vermeintlich nicht therapierbare Straftäter in den Niederlanden neue Impulse. ${ }^{11}$ Ihre Eignung als menschenfreundliche Alternative zur Sicherungsverwahrung wird im Folgenden erörtert.

\section{Die Einführung von Longstay-Einrichtungen in den Niederlanden}

Das niederländische Rechtsfolgensystem ist wie auch das deutsche Recht ein zweispuriges. ${ }^{12}$ Wie in Deutschland wird zwischen Strafen und Maßregeln der Besserung und Sicherung unterschieden. Eine mit der Sicherungsverwahrung vergleichbare Maßregel gibt es in den Niederlanden heute nicht. Pläne, die Sicherungsverwahrung 1929 einzuführen, wurden auf Grund rechtsstaatlicher Bedenken verworfen. ${ }^{13}$ Auf den in Deutschland in die Sicherungsverwahrung kommenden Personenkreis wird in den Niederlanden einerseits mit Präventionsbelange berücksichtigenden erhöhten Strafen und andererseits mit der Maßregel der Überlassung mit Versorgung von Staats wegen gegenüber psychisch gestörten Straftätern reagiert. Hierbei handelt es sich um eine mit dem psychiatrischen Krankenhaus nach \63 StGB vergleichbare behandlungsorientierte Maßregel, die allerdings auch die Gruppe der persönlichkeitsgestörten, aber nicht kranken Straftäter erfasst. ${ }^{14}$ Bei der Klientel, die für die Unterbringung in den Longstay-Einrichtungen in Betracht kommt, handelt es sich um Personen, die zunächst in mit dem deutschen psychiatrischen Krankenhaus vergleichbaren Einrichtungen des Maßregelvollzugs untergebracht worden sein müssen.

Die Gründe der Einführung von Longstay-Konzepten in den Niederlanden sind sehr unterschiedlich: Der Maßregelvollzug leidet dort - wie auch in Deutschland ${ }^{15}$ - unter einem massiven Anstieg der Zahl der Untergebrachten. Er sieht sich mit einer personell und finanziell immer schwieriger werdenden Situation konfrontiert. Diese Ausgangssituation hat die Niederländer veranlasst, neue Wege im Umgang mit einer Klientel zu beschreiten, welche die therapeutische Arbeit im Maßregelvollzug zusätzlich belastet. Hierbei handelt es sich um Personen, die sich bereits seit langem im Maßregelvollzug befinden und deren Gefährlichkeit trotz intensiver therapeutischer Hilfe weiter besteht. Einige dieser Patienten weigern sich nicht nur, selbst an den therapeutischen Angeboten teilzunehmen, sondern versuchen, die Arbeit in den psychiatrischen Einrichtungen zu torpedieren, wodurch die Arbeit mit den therapiewilligen Patienten deutlich erschwert wird und Therapieplätze durch therapieunwillige Patienten blockiert werden. ${ }^{16} \mathrm{Da}$ bei dieser Personengruppe z.T. eine hohe Rückfallgefahr besteht, können die psychiatrischen Einrichtungen die "Störer" nicht einfach in die Freiheit entlassen. Die in den Niederlanden eingeführten Longstay-Einrichtungen sollen einen Ausweg aus diesem Dilemma darstellen und den Maßregelvollzug von dieser Personengruppe entlasten ${ }^{17}$ und nicht zuletzt die finanziellen Aufwendungen für diese vermeintlich „hoffnungslosen Fälle“ reduzieren. ${ }^{18}$ Die Forderung nach dauerhafter Unterbringung „unverbesserlicher" Straftäter in Longstay-Abteilungen ist nicht zuletzt auf einen wachsenden therapeutischen Nihilismus zurückzuführen, dessen Vertreter ausschließlich in negativer Spezialprävention einen hinreichenden Schutz der Allgemeinheit vor neuen Straftaten erblikken. ${ }^{19}$ Es spielen somit nicht allein, womöglich sogar nicht einmal in erster Linie, Überlegungen eine Rolle, wie eine Humanisierung der Unterbringungsbedingungen für rückfallgefährdete Straftäter zu erreichen wäre, sondern vorwiegend Überlegungen, wie die Kapazitätsprobleme im Maßregelvollzug zu lösen seien.

Nichtsdestotrotz sollte überlegt werden, ob Longstay-Konzepte eine wünschenswerte, verfassungsrechtlich zulässige Alternative zur Sicherungsverwahrung darstellen können.

Die Unterbringung von vermeintlich nicht therapierbaren Personen aus dem Maßregegelvollzug in Longstay-Einrichtungen erfolgt in den Niederlanden, wenn zwei ernsthafte Behandlungsversuche von jeweils drei Jahren Dauer in zwei unterschiedlichen Einrichtungen des Maßregelvollzugs fehlgeschlagen sind, d.h. eine weitere Deliktsgefahr besteht und das höchste Berufungsgericht für Maßregelpatienten in den Niederlanden die vermeintlich nicht behebbare Gefährlichkeit der Personen ebenfalls bejaht hat. ${ }^{20}$ Liegen diese Voraussetzungen vor, wird die Person in die Longstay-Anstalt verlegt.

\section{Lebensbedingungen in Longstay-Einrichtungen}

Um beurteilen zu können, ob die Longstay-Einrichtungen eine wünschenswerte Alternative zur Sicherungsverwahrung darstellen, sind die Lebensbedingungen in den Anstalten vorzustellen. ${ }^{21}$ Beispielhaft sollen hierfür die Bedingungen in der Longstay-Einrichtung im Forensisch Psychiatrischen Zentrum Veldzicht in Balkbrug, die als Pilotprojekt mit 20 Plätzen im April 1999 ihre Arbeit aufgenommen hat, dargestellt werden. Bemerkenswert sind zunächst die örtlichen und baulichen Gegebenheiten, die sich deutlich von den Bedingungen im deutschen Sicherungsverwahrungsvollzug unterscheiden. Auf dem Land sind auf einem großen Gelände zwei Pavillons errichtet worden. Die Bewohner leben in einer Art Dorfgemeinschaft zusammen. Innerhalb des Geländes wird ihnen eine möglichst große Autonomie und Bewegungsfreiheit eingeräumt. Dazu wurden frei zugängliche Küchen und Aufenthaltsräume geschaffen. Es wird versucht, die bestehenden Fähigkeiten und Hobbys zu fördern. So können die Bewohner etwa ein Gartenstück anlegen oder in einem an das Anstaltsgelände angrenzenden Kanal angeln. Die persönlichen Freiheiten sollten allerdings auch nicht überschätzt werden: So ist der Konsum von Alkohol und selbst das einverständliche Ausleben sexueller Bedürfnisse nicht gestattet. Für arbeitsfähige Bewohner besteht eine Arbeitspflicht von wöchentlich dreißig Stunden. ${ }^{22}$

Therapeutische Hilfe, die darauf abzielt, die Bewohner auf ein Leben außerhalb der Anstalt vorzubereiten und ihre Gefährlichkeit zu beseitigen, findet in den Einrichtungen nicht statt. ${ }^{23}$

Das erklärte Ziel der betreuerischen Arbeit ist nämlich nicht die erfolgreiche Therapie der Bewohner, sondern die möglichst große Förderung des Wohlbefindens in der Einrichtung. ${ }^{24}$ Soweit therapeutische Hilfe gewährt wird, besteht diese in erster Linie in dem Versuch, bei den Untergebrachten Akzeptanz für ihre voraussichtlich dauerhafte Unterbringung zu vermitteln. Perik, Mitarbeiter der Direktion der Longstay-Einrichtung, erklärt ausdrücklich, das Ziel der therapeutischen Arbeit bestehe maßgeblich in der „Hospitalisierung“ der Bewohner, woraus auch gegenüber den Betroffenen kein Hehl gemacht wird. ${ }^{25}$ Am Anfang der Unterbringung werden diese mit ihrer neuen persönlichen Situation konfrontiert. Unmissverständlich wird ihnen mitgeteilt, dass sie mit hoher Wahrscheinlichkeit den Rest ihres Lebens in der Anstalt verbringen werden. Eine solche Mitteilung ist für viele der Untergebrachten ein Schock. Die Einrichtun- 
gen versuchen, diese psychische Belastung durch sog. Trauerarbeit abzumildern. ${ }^{26}$ Dies scheint aber nur bedingt zu gelingen. Wie sich diese Situation auf die psychische Verfassung der Untergebrachten auswirkt, tritt bereits jetzt offen zu Tage: Die Bewohner der Einrichtungen zeigen erhebliche Gefühlsschwankungen. Einige werden phasenweise aktiv und sind gut gelaunt, dann fallen sie wiederum in depressive Zustände mit großer Inaktivität. Auf Zeiten der Kooperation folgen wieder Perioden, in denen sich die Bewohner gegenüber den Einrichtungsmitarbeitern aggressiv verhalten. ${ }^{27}$

Ein Suizid und drei Entweichungen aus dem Urlaub zeigen deutlich, dass zumindest einige der Untergebrachten massiv unter ihrer hoffnungslosen Situation leiden. ${ }^{28}$ Wiederum andere Bewohner empfinden das Abfallen des Therapiedrucks als Befreiung. ${ }^{29}$ Doch auch diejenigen, die sich den ersten Berichten zu Folge mit der Situation in der Einrichtung arrangiert zu haben scheinen, äußern die Hoffnung, zukünftig in eine therapeutische Einrichtung zurückverlegt zu werden. ${ }^{30}$

Die Möglichkeit, die Freiheit wieder zu erlangen, ist nicht gänzlich verschlossen: Die Longstay-Einrichtungen sehen eine regelmäßige im Abstand von zwei Jahren vorgenommene Überprüfung des Weiterbestehens der Gefährlichkeit vor. Die Chance entlassen zu werden, dürfte aber ausgesprochen gering sein, da therapeutische Maßnahmen, die auf den Abbau der Rückfallgefahr gerichtet sind, praktisch nicht stattfinden. ${ }^{31}$ Eine Rückverlegung in den therapeutischen Vollzug auf den „bloßen Wunsch“ der Betroffenen ist nach dem niederländischen Longstay-Konzept nicht vorgesehen. Rückverlegungen kommen nur in Betracht, wenn neue Umstände Anlass geben, die therapeutischen Möglichkeiten bei der konkreten Person anders zu beurteilen. ${ }^{32}$ Durch welche Umstände aber eine solche Verhaltensänderung angesichts nicht stattfindender therapeutischer Angebot geschehen soll, ist nicht ersichtlich.

\section{Die verfassungsrechtliche Bewertung von Longstay- Konzepten}

Bei der verfassungsrechtlichen und rechtspolitischen Bewertung von Longstay-Konzepten ist ein Hauptaugenmerk darauf zu legen, ob die Einrichtungen eine Verbesserung der unzureichenden Vollzugssituation der Sicherungsverwahrten bewirken. Von der unzureichenden Anhebung des Unterbringungskomforts der Sicherungsverwahrten in Deutschland unterscheidet sich die Ausstattung der LongstayEinrichtungen erheblich. Für die Untergebrachten bedeuten sie eine Liberalisierung der Vollzugsbedingungen, die aus Sicht der Sicherungsverwahrten in Deutschland eine wünschenswerte Verbesserung darstellen und dem verfassungsrechtlichen Abstandsgebot Rechnung tragen würde.

Gegen Longstay-Konzepte werden allerdings auch erhebliche Bedenken vorgebracht. Eine große Schwierigkeit solcher Ansätze besteht bereits darin, die Personen zu identifizieren, denen mit den heute bestehenden therapeutischen Möglichkeiten nicht geholfen werden kann. ${ }^{33}$ Eine neuere Untersuchung Leygrafs dokumentiert, dass Maßregelpatienten in Deutschland nicht selten vorschnell als „unverbesserlich “ etikettiert werden. Der Anteil der als nicht therapierbar eingestuften Patienten differierte in den Einrichtungen zwischen 0 und $35,7 \% .{ }^{34}$ Dies lässt darauf schließen, dass die Frage der „Untherapierbarkeit“ in hohem Maße von Wertungen des Personals der Einrichtungen abhängig ist. ${ }^{35}$ „Therapieresistenz “ kann im Übrigen auch auf unzureichende therapeutische Angebote zurückzuführen sein. Lösel bezeichnet „Unbehandelbarkeit“ treffend als „Interaktionsergebnis“ zwischen dem Klienten und dem therapeutischen Hel- fer. ${ }^{36}$ Bei der Frage der Unbehandelbarkeit befindet sich die Wissenschaft weiterhin auf einem „unsicheren Terrain. “Weitere Forschung ist dringend erforderlich. ${ }^{37}$ Bei Personengruppen, die lange Zeit als nicht therapierbar galten, zeichnen sich durch die Entwicklung neuer Behandlungskonzepte neue Möglichkeiten ab. ${ }^{38}$ Erfahrungen der Strafrechtspraxis zeigen, dass „nahezu alle der ,gefährlichen’ und hochbelasteten Täter früher oder später Resozialisierungsangebote annehmen und nicht mehr rückfällig werden. “39 Die meisten Sicherungsverwahrten sind nicht dauerhaft gefährlich. So zeigt die neuere Untersuchung Kinzigs, dass die meisten Personen nach einigen Jahren nach $\$ 67 \mathrm{~d}$ Abs. 2 StGB wieder aus dem Vollzug der Sicherungsverwahrung entlassen werden. ${ }^{40}$ Wie ist mit einem solchen Befund umzugehen? Die Personen, bei denen die formellen und materiellen Voraussetzungen der Sicherungsverwahrung vorliegen, pauschal als „unverbesserlich“ einzustufen, wird der Realität nicht gerecht.

Ob Longstay-Einrichtungen nach dem niederländischen Vorbild eine rechtlich zulässige Alternative zum Vollzug der Sicherungsverwahrung darstellen, ist äußerst zweifelhaft, da in ihrem Vollzug praktisch keine Angebote gemacht werden, welche die Bewohner zu einem Leben in Freiheit befähigen sollen. Ob solche Anstalten verfassungsgemäß sind, hängt davon $a b$, ob die Untergebrachten ein freiwilliges Rückkehrrecht in den therapeutischen Vollzug haben. Im niederländischen Modell werden die Bewohner gegen ihren Willen in die Longstay-Einrichtungen gebracht und dort womöglich lebenslang verwahrt. Vollzugskonzepte, die sich auf die sichere Verwahrung der Untergebrachten beschränken, geben den Betroffen als Menschen auf und behandeln ihn nur noch analog einer gefährlichen Sache. Daran können auch einige Liberalisierungen des Vollzugs der Maßregel nichts ändern. ${ }^{41}$ Das zentrale Bedürfnis eines gefangenen Menschen ist die Wiedererlangung seiner Freiheit. Das $B$ VerfG hat deshalb zu Recht festgestellt, dass der Vollzug der Sicherungsverwahrung neben dem verfassungsrechtlichen Abstandsgebot so ausgerichtet werden muss, dass die Untergebrachten eine Chance haben, ihre Freiheit wiederzuerlangen. Anderenfalls verstößt der Vollzug gegen die menschliche Würde (Art. 1 Abs. 1 GG). ${ }^{42}$ Dieser Grundsatz muss auf die Longstay-Einrichtungen übertragen werden. Ein reiner Verwahrvollzug für vermeintlich „Unverbesserliche“, den die Untergebrachten nicht auf eigenen Wunsch wieder verlassen dürfen, ist verfassungsrechtlich unzulässig. ${ }^{43}$

$\mathrm{Zu}$ prüfen ist, ob eine Unterbringung in den Longstay-Einrichtungen im Falle der Zustimmung der Untergebrachten zulässig sein könnte. Lindemann hält eine solche Einwilligung angesichts der Tragweite des Entschlusses für unwirksam. Sie habe den Charakter eines unzulässigen Grundrechtsverzichts. ${ }^{44}$ Hiergegen wird von Befürwortern von Longstay-Konzepten eingewandt, dass eine solche Sicht den Untergebrachten weiter entmündige und auf eine Einschränkung der freien Willensbildung der Betroffenen hinauslaufe. ${ }^{45}$

Lindemann ist mit seiner kritischen Bewertung der Zustimmungslösung im Falle einer Ausgestaltung nach niederländischem Recht aber beizupflichten. Werden Verwahrte unter dem Eindruck der hoffnungslosen Situation im Vollzug der Sicherungsverwahrung mit der Möglichkeit konfrontiert, unwiderruflich in eine andere Anstalt zu wechseln, werden viele von ihnen vorschnell für die Verlegung in die Longstay-Einrichtung plädieren, ihre Entscheidung aber womöglich bereuen. Sie befinden sich in einer Situation, die Amelung treffend als „Einwilligung des Unfreien“ charakterisiert hat. ${ }^{46}$ Selbst wenn man davon ausgeht, dass Personen in einer solchen Situation die Fähigkeit besäßen, sich frei zu entscheiden, ist aus verfassungsrechtlichen Gründen eine Zustimmung zur niederländischen Konzeption 
für unbeachtlich zu halten. Dem Wunsch, nicht an Therapieangeboten teilnehmen zu wollen, dauerhaft zu entsprechen, würde der sozialstaatlichen Verpflichtung des Staates, für die Wiedereingliederung hilfsbedürftiger Straftäter zu sorgen, nicht gerecht. Weil das bloße Wegsperren mit der Menschenwürde der Person unvereinbar ist, stellt sich im Übrigen die Frage, ob eine Person in eine solche Behandlung überhaupt einwilligen kann. Dies ist zu verneinen, da auch die Grundrechtsträger nicht über ihre Menschenwürde disponieren können. ${ }^{47}$ Das niederländische Longstay-Konzept kann daher aus verfassungsrechtlicher Sicht nicht als Alternative zur Sicherungsverwahrung fungieren.

Als verfassungsgemäß wären allenfalls Einrichtungen anzusehen, in welche die Sicherungsverwahrten auf freiwilliger Basis wechseln und bei denen sich die Untergebrachten, wenn eine Bereitschaft zur Wiederaufnahme von Therapiemaßnahmen besteht, in den therapeutischen Vollzug zurückverlegen lassen könnten. ${ }^{48}$ Ein solches Konzept könnte als „time out“ für die Untergebrachten eine Möglichkeit sein, noch einmal Kraft für einen erneuten Therapieversuch zu schöpfen. Auch hier besteht freilich die sozialstaatliche Aufgabe, die Untergebrachten zur Wiederaufnahme einer Therapie zu motivieren. ${ }^{49}$ Fraglich ist allerdings, ob für eine solche Konzeption überhaupt sog. Longstay-Einrichtungen erforderlich sind. Nach der hier vertretenen Auffassung besteht ein solches Bedürfnis nicht. Menschen, die sich im Vollzug freiheitsentziehender Sanktionen befinden, haben auch im therapeutischen Vollzug das Recht und die Möglichkeit, therapeutische Angebote abzulehnen. Die Argumente, die von den Befürwortern der Longstay-Einrichtungen gegen die verfassungsrechtliche Kritik vorgebracht werden, können auch in ihrer Entgegensetzung - Komfort innerhalb der Einrichtung versus therapeutische Angebote - nicht überzeugen. Weshalb es nicht möglich sein soll, Einrichtungen zu schaffen, die beiden verfassungsrechtlichen Vorgaben, der Resozialisierungsidee und dem verfassungsrechtlichen Abstandsgebot entsprechen, ist nicht verständlich.

\section{Zusammenfassung}

Longstay-Einrichtungen bedeuten aus der Sicht der Betroffenen gegenüber dem Vollzug der Sicherungsverwahrung eine Verbesserung der Aufenthaltsmöglichkeiten im Freiheitsentzug. Eine Alternative zur Sicherungsverwahrung stellen sie allerdings nicht dar. Freiheitsentzug, der in seiner Ausgestaltung nicht auf die Wiedererlangung von Freiheit gerichtet ist, ist mit der Menschenwürde des Art. 1 Abs. 1 GG nicht vereinbar. Menschen, die sich im Vollzug freiheitsentziehender Sanktionen befinden, haben das Recht, Resozialisierungsangebote abzulehnen und sich im therapeutischen Vollzug eine Auszeit zu nehmen. Zur Wahrung dieses Rechts und zur Realisierung des verfassungsrechtlichen Abstandsgebots zwischen dem Vollzug der Freiheitsstrafe und freiheitsentziehender Maßregeln werden Longstay-Einrichtungen nicht benötigt. Diese verfassungsrechtlichen Vorgaben lassen sich vielmehr auch in therapeutischen Vollzugskonzeptionen gewährleisten. ${ }^{50}$

\section{Verwendete Literatur:}

Albrecht, Peter-Alexis: Das nach-präventive Strafrecht, in: Institut für Kriminalwissenschaften und Rechtsphilosophie Frankfurt a.M. (Hrsg.): Jenseits des rechtsstaatlichen Strafrechts, Frankfurt a.M. 2007, S.3.

Amelung, Knut: Die Einwilligung der Unfreien, ZStW 95 (1983), 1.

Barton, Stephan: Prognosegutachten, Neurobiologie, Sicherungsverwahrung, in: Ders. (Hrsg.): „... weil er für die Allgemeinheit gefährlich ist! “, BadenBaden 2006, S.11.

Baumann, Jürgen: Was würde uns die Strafrechtsreform bringen?, in: Ders.: Kleine Streitschriften zur Strafrechtsreform, Bielefeld 1965, S.14.
Bielefeldt, Heiner: Menschenrechte in der Einwanderungsgesellschaft, Bielefeld 2007.

Böhm, Alexander: 25 Jahre Strafvollzugsgesetz, BewHi 2002, 92.

Böllinger, Lorenz: Gefährlichkeit als iatrogene Krankheit - Sicherungsverwahrung ohne Grenzen, in: Burckhardt, Sven-U. u.a. (Hrsg.): Korrespondenzen (= Festschrift für Feest), Münster 2005, S.138.

Braasch, Matthias: Untherapierbare Straftäter im Maßregelvollzug, Mönchengladbach 2006.

Dessecker, Axel: Die Überlastung des Maßregelvollzugs, Neue Kriminalpolitik 2005, 23.

Dünkel, Frieder: Aktuelle Daten zur Sicherungsverwahrung, Forum Strafvollzug 2008,76 .

Dünkel, Frieder / Maelicke, Bernd: Irren ist (un-) menschlich!, Neue Kriminalpolitik 2004, 131.

Eisenberg, Ulrich: Kriminologie, 6. A. München 2005.

- Die Maßregel der Unterbringung in einem psychiatrischen Krankenhaus gem. $\$ 63$ StGB und so genannte „Nicht-Therapiegeeignetheit“, NStZ 2004, 240.

Frommel, Monika: „Eene meene muh - drin bist Du“ - Zur Wiederkehr der Sicherungsstrafe in der gegenwärtigen Diskussion, KJ 1995, 226.

Haffke, Bernhard: „Unbehandelbare“ Straftäter in: Rehn, Gerhard u.a. (Hrsg.): Freiheit und Unfreiheit. Arbeit mit Straftätern außerhalb und innerhalb des Justizvollzuges, Herbolzheim 2004, S.78.

Kant, Immanuel: Über den Gemeinspruch: Das mag in der Theorie richtig sein, taugt aber nicht für die Praxis, 1793, Akademie Ausgabe Berlin 1902, S.273.

Kiesel, Manfred: Echo zu: Hefendehl / Hohmann, ZRP 2001, 23: Fischen im Trüben: Christlich-Demokratische Kriminalpolitik 2001, 385.

Kinzig, Die Sicherheitsverwahrung auf dem Prüfstand, Freiburg 1996.

Kobbé. Ulrich: „No cure, but control“ - Resozialisierungsunwillige und therapieresistente Straftäter als Adressaten des Zwangs heute, in: Nickolai, Werner/ Reindl, Richard (Hrsg.): Renaissance des Zwangs - Konsequenzen für die Straffälligenhilfe, Freiburg 1999, S.71.

Köhler, Michael: Die Aufhebung der Sicherungsmaßregeln durch die Strafgerechtigkeit, in: Pawlik, Michael u.a. (Hrsg.): FS für Jakobs, Berlin 2007, S.273.

Köhne, Michael: Resozialisierungsunfähige Strafgefangene, ZRP 2003, 207.

Kohlrausch, Eduard: Sicherungshaft. Eine Besinnung auf den Streitstand, ZStW 44 (1924), 21.

Kreuzer, Arthur: Zur Entwicklung äußerster kriminalpolitischer Instrumente - Todesstrafe, Folter, lebenslange Freiheitsstrafe und Sicherungsverwahrung in Deutschland, in: Schüler-Springorum, Horst \& Nedopil, Norbert (Hrsg.) FS für Hisao Katoh, Lengerich 2008, S.61.

Kreuzer, Arthur \& Bartsch, Tillmann: Gesetzgeberische Flickschusterei und Vollzugsprobleme bei der Sicherungsverwahrung, Forum Strafvollzug 2008, 30 .

Kunz, Karl-Ludwig: Die Sicherung als gefährlich eingestufter Rechtsbrecher, in: Barton, Stephan (Hrsg.): ,...weil er für die Allgemeinheit gefährlich ist! “, Baden-Baden 2006, S.71.

Laubenthal, Klaus: Die Renaissance der Sicherungsverwahrung, ZStW 116 (2004), 703.

Leygraf, Norbert: Verschiedene Möglichkeiten als nicht therapierbar zu gelten, R \& P 2002, 3.

Lindemann, Michael: Nachträgliche Sicherungsverwahrung - Jenseits von Strafvollzug und Maßregelvollzug? Konsequenzen für eine menschenwürdige Unterbringung, Vortragsmanuskript 2005.

- Zur Vereinbarkeit gesonderter Longstay-Abteilungen im Maßregelvollzug mit den geltenden (verfassungs-) rechtlichen Vorgaben, R \& P 2002, 8 .

Die Behandlung der Unbehandelbaren, R \& P 2001, 21.

Lösel, Friedrich: „Unbehandelbare“ Straftäter: Probleme und Lösungsansätze, in: Rehn, Gerhard (Hrsg.), Freiheit und Unfreiheit. Arbeit mit Straftätern innerhalb und außerhalb des Justizvollzuges, Herbolzheim 2004, S.368.

Lüderssen, Klaus: Die ewige Versuchung des Täterstrafrechts, KJ 2006, 361.

Martens, Willem: What shall we do with untreatable forensic psychiatrie patients?, Med Law 2000, 389.

Müller-Dietz, Heinz: Unterbringung in der Entziehungsanstalt und Verfassung, JR 1995, 353.

Mushoff, Tobias: Strafe - Maßregel - Sicherungsverwahrung. Eine kritische Untersuchung über das Verhältnis von Schuld und Prävention, Verlag Peter Lang, Frankfurt a.M. 2008.

Naucke, Wolfgang: Strafrecht, 10. Auflage, Neuwied 2002.

Nedopil, Norbert: „Unbehandelbare“ Straftäter: Kehrseite des Behandlungsfortschritts, in: Rehn, Gerhard u.a. (Hrsg.): Freiheit und Unfreiheit. Arbeit mit Straftätern innerhalb und außerhalb des Justizvollzuges, Herbolzheim 2004, S.356.

Nuhn-Naber, Carmen / Rehder, Ulrich / Wischka, Bernd: Behandlung von Sexualstraftätern mit kognitiv-behavioralen Methoden: Möglichkeiten und Grenzen, MschrKrim 2002, 271.

Nuhn-Naber, Carmen / Rehder, Ulrich: Psychopathie - Gegenindikation für Sozialtherapie?, MschrKrim 2005, 257. 
Osterheider, Michael: Long-Stay-Abteilungen - Vorbedingungen, Möglichkeiten und Begrenzungen, R \& P 2002, 17.

Perik, J.C.A.: Longstay in der Praxis, R \& P 2002, 23.

Pfäfflin, Friedemann: Drei Fragen zum Maßregelvollzug, gestellt von außen, in: Duncker, Heinfried u.a. (Hrsg.): Ulrich Venzlaff zum 85. Geburtstag, Lengerich u.a. 2006, S.266.

Pollähne, Helmut: Endstation Unrechtsstaat? Mit der Sicherungsverwahrung auf Zeitreise, in: Komitee für Grundrechte und Demokratie, Jahrbuch 2008, Münster 2008, S.122.

- Trendwende im Strafrecht? - Aktuelle Entwicklungen im Maßregelrecht unter besonderer Berücksichtigung der Sicherungsverwahrung, SchlHA 2005,135

- Wenn Patienten in „long stay units“ (ver-) enden, in: Müller-Heidelberg, Till u.a. (Hrsg.): Grundrechte-Report 2005, Frankfurt a.M. 2005, S.195.

Rasch, Wilfried / Konrad, Norbert: Forensische Psychiatrie, 3. Auflage, Stuttgart 2004.

Roth, Reinhold: „Unbehandelbare“ Straftäter aus strafvollstreckungsrechtlicher Sicht, in: Rehn, Gerhard u.a. (Hrsg.): Freiheit und Unfreiheit, Herbolzheim 2004, S.94.

Rzepka, Dorothea: Sicherheits- statt Rechtsstaat - Überblick und Anmerkungen zu bundes- und landesrechtlichen Konzepten einer nachträglichen Sicherungsverwahrung, Teil 1 und 2, Recht \& Psychiatrie 2003, 127, 191.

Sagel-Grande, Irene: Modernisierung des Sanktionssystems und der Sanktionsanwendung in den Niederlanden, MschrKrim 2006, 427.

- Die Maßregeln der Besserung im strafrechtlichen Rechtsfolgensystem der Niederlande, ZStW 103 (1991), 732.

- Longstay, in: Barton, Stephan (Hrsg.): ,...weil er für die Allgemeinheit gefährlich ist! “, Baden-Baden 2006, S.187.

Schott, Martin: Wie offen ist der Maßregelvollzug heute?, in: Duncker, Heinfried u.a. (Hrsg.): Ulrich Venzlaff zum 85. Geburtstag, Lengerich u.a. 2006, S.346.

Schwind, Hans-Dieter: Strafvollzug in der Konsolidierungsphase, ZfStrVo 1988, 259.

Schubert, Karin: Zur Eröffnung der Sozialtherapeutischen Anstalt - Bereich 2 - der Justizvollzugsanstalt Tegel, ZfStrVo 2003, 259.

Streng, Franz: Das Legitimations-Dilemma sichernden Freiheitsentzugs - Überlegungen zur neueren Rechtsentwicklung, in: Dölling, Dieter (Hrsg.): FS für Lampe, Berlin 2003, S.611.

Sturm, Gerd: Probleme eines Verzichts auf Grundrechte, in: Leipholz, Gerhard (Hrsg.): FS für Willi Geiger, Tübingen 1974, S.173

Tiedemann, Klaus: Verfassungsrecht und Strafrecht, Heidelberg 1991.

Volckart, Bernd / Grünebaum, Maßregelvollzug, 6. Auflage, Neuwied 2003.

\section{Fußnoten}

$1^{*}$ Der vorliegende Beitrag fußt auf Überlegungen meiner 2008 ersch. Dissertation: Strafe - Maßregel - Sicherungsverwahrung.

2 Vgl. Dünkel, Forum Strafvollzug 2008, 76 ff.

3 Kreuzer \& Bartsch, Forum Strafvollzug 2008, 30 (31).

4 BVerfGE 109, 133; BVerfGE 109, 190; BVerfG, NStZ 2006, 574.

5 S. exemplarisch aus jüngerer Zeit: P. A. Albrecht in: Institut für Kriminalwissenschaften und Rechtsphilosophie Frankfurt a.M., Jenseits des rechtsstaatlichen Strafrechts, S.10, 14 f.; Barton in: Ders. (Hrsg.), ...weil er für die Allgemeinheit gefährlich ist, S.26; Böllinger, FS für Feest, S.138; Fischer, StGB, $\int 66$ Rn. 3; Köhler, FS für Jakobs, S.273; Kreuzer, FS für Hisao Katoh, S.77 ff.; Kunz in: Barton (Hrsg.), a.a.O., S.82 ff.; Laubenthal, ZStW 116 (2004), 703; Lüderssen, KJ 2006, 361; Mushoff, a.a.O., S.305 ff.; Naucke, Strafrecht, S.99 f.; Pollähne in: Komitee für Grundrechte und Demokratie (Hrsg.), Jahrbuch 2008, S.122; Rzepka, R \& P 2003, 127 ff., 191 ff.; Streng, FS für Lampe, S.611 und bereits: Frommel, KJ 1995, 226 (230); Kinzig, Die Sicherungsverwahrung auf dem Prüfstand, S.597 ff.

6 Vgl. Mushoff, a.a.O., S.252 ff.

7 Kohlrausch, ZStW 44 (1924), 33.

8 Dazu ausführlich: Mushoff, a.a.O., S.311 ff.; s.a. Kreuzer \& Bartsch, Forum Strafvollzug 2008, $30 \mathrm{ff}$.

9 Vgl. einerseits BVerfGE 2, 118 und andererseits BVerfGE 109, 133.

10 Baumann, Kleine Streitschriften zur Strafrechtsreform, S.24; Eisenberg, Kriminologie, $\mathbb{S} 34$ Rn. 65; Tiedemann, Verfassungsrecht und Strafrecht, S.33.

11 Zur Diskussion, ob Longstay-Einrichtungen als Alternative zur Sicherungsverwahrung in Betracht kommen, bereits: Barton in: Ders. (Hrsg.), Fn. 4 , S.26 f.; Lindemann, Nachträgliche Sicherungsverwahrung - Jenseits von Strafvollzug und Maßregelvollzug?, S.1 ff.; vgl. zur deutschen Diskussion über den Umgang mit nicht therapiewilligen bzw. vermeintlich nicht the- rapierbaren Strafgefangenen und Patienten im Maßregelvollzug: Böhm, BewHi 2002, 92, 101 f.; Braasch, Untherapierbare Straftäter im Maßregelvollzug, 2006; Eisenberg, NStZ 2004, 240; Kobbé in: Nicolai/ Reindl (Hrsg.), Renaissance des Zwanges, 1999, S.71; Köhne, ZRP 2003, 207; Leygraf, R \& P 2002, 3; Osterheider, R \& P 2002, 17 (17) sowie: Haffke, Lösel, Nedopil und Roth in: Rehn u.a. (Hrsg.), Freiheit und Unfreiheit, 2004, S. 78 ff., 94 ff., 356 ff., 368 ff.; Nuhn-Naber u.a., MschrKrim 2002, 271, 277 f.; Dies./ Rehder, MschrKrim 2005, 257; Pollähne, GrundrechteReport 2005, S.195; s.a. Martens, Med Law 2000, 389 ff.

12 Vgl. Sagel-Grande, ZStW 103 (1991), 732, 739 f.; Dies. in: Barton (Hrsg.), Fn. 4, S.187 ff.

13 Kinzig, Fn. 4, S.510 f.; Sagel-Grande, MschrKrim 2006, 427 (437).

14 Lindemann, Fn. 10, S.4.

15 S. Dessecker, Neue Kriminalpolitik 2005, 23 ff.

16 Rasch / Konrad, Forensische Psychiatrie, 2004, S.106; Volckart / Grünebaum, Maßregelvollzug, S.106.

17 Lindemann R \& P 2001, 21.

18 Braasch, Fn. 9, S.495 ff., 527 ff.; Perik, R \& P 2002, 23 (25); solche Forderungen werden auch in Deutschland immer wieder erhoben, z.B. von Kiesel, ZRP 2001, 385 (386); Schwind, ZfStrVo 1988, 259 (261).

19 Leygraf, R \& P 2002, 3 (4).

20 Perik, R \& P 2002, 23 (23).

21 Vgl. zum Folgenden besonders: Braasch, Fn. 10, S.553 ff.; Lindemann, Fn. 10, S. 4 ff.; Ders., R \& P 2001, 21 ff.; Ders., R \& P 2002, 88 ff.; Perik, R \& P 2002, 23 ff.; Sagel-Grande, MschrKrim 2006, 427, 437 ff.

22 Perik, R \& P 2002, 23 (26).

23 Perik, R \& P 2002, 23 (26).

24 Vgl. Volckart / Grünebaum, Fn. 15, S.224.

25 Perik, R \& P 2002, 23 (26).

26 Braasch, Fn. 10, S.557 f.; Perik, R \& P 2002, 23 (25).

27 Vgl. Perik, R \& P 2002, 23 (26).

28 Lindemann, Fn. 10, S.6.

29 Volckart / Grünebaum, Fn. 15, S.224.

30 Perik, R \& P 2002, 23 (25).

31 Lindemann, Fn. 10, S.5.

32 Perik, R \& P 2002, 23 (26).

33 Osterheider, R \& P 2002, 17 (18).

34 Leygraf, R \& P 2002, 3 (3).

35 Köhne, ZRP 2003, 207 (208).

36 Lösel in: Rehn u.a. (Hrsg.), Fn. 10, S.379.

37 Lösel in: Rehn u.a. (Hrsg.), Fn. 10, S.369, 376.

38 Lösel in: Rehn u.a. (Hrsg.), Fn. 10, S.368 ff.

39 Dünkel / Maelicke, Neue Kriminalpolitik 2004, 131; vgl. auch: Martens, Med Law 2000, 389 (390); Müller-Dietz. JR 1995, 353 (359); Schubert, ZfStrVo 2003, 259.

40 Kinzig, Fn. 4, S.457.

41 Lindemann, Fn. 10, S.6; krit. a. Pfäfflin, FS für Venzlaff zum 85. Geb., 2006, S.278; Pollähne, SchlHA 2005, 135 (138).

42 BVerfGE 109, 133, 150 ff.

43 Lindemann, Fn. 10, S.10; Ders., R \& P 2001, 21 (27); Pollähne, Grundrechte-Report 2005, S.195; Roth in: Rehn u.a. (Hrsg.), Fn. 10, S.101, der die Longstay-Einrichtungen pointiert als „Horrorkabinett“ bezeichnet.

44 Lindemann R \& P 2002, 8, 14 f.

45 Volckart / Grünebaum, Fn. 15, S.225.

46 Amelung, ZStW 95 (1983), 1, bes. S.9 f.

47 Bielefeldt, Menschenrechte in der Einwanderungsgesellschaft, S.29 f.; Sturm, FS für Geiger, S.173 (189); i.E. wie hier: Seelmann, Rechtsphilosophie, $\mathbb{S} 12$ Rn. 14 und bereits: Kant, Werke Akademie Ausgabe Bd. VIII, S.304.

48 Vgl. auch: Osterheider, R \& P 2002, 17, 19 f.

49 Vgl. auch: Schott, FS für Venzlaff zum 85. Geb., S.350. Zutreffend weist Lösel in: Rehn u.a. (Hrsg.), Fn. 10, S.372 f., darauf hin, dass die Dichotomie Behandlungswilligkeit bzw. -fähigkeit und Behandlungsresistenz viel zu „grob“ ist. Ambivalente Erwartungen der Betroffenen sind bei therapeutischen Maßnahmen die Regel und nicht die Ausnahme. Als Ansatz stellt Lösel, ebd., S.379, das „Motivational Interview“ vor. Hierbei geht es besonders darum, in einem Dialog „selbst-motivierende Äußerungen des Probanden “ $\mathrm{zu}$ fördern.

50 S. zu den Potentialen eines umfassenden sozialtherapeutischen Gesamtkonzeptes nach dem Vorbild der Maßregellösung des $\mathbb{6} 65$ StGB a.F.: Mushoff, a.a.O., S.499 ff. 